\title{
UTILIDAD DEL SCORE SOFA EN LA PREDICCIÓN DE MUERTE MATERNA EN LA UCI MATERNA DEL INMP
}

\author{
Walter J. De la Peña Meniz ${ }^{1, a}$, Alberto Diaz Seminario, ${ }^{1, a}$, Ronald Meza Salcedo, ${ }^{1, a}$, Hernan Sandoval Manrique ${ }^{1, a}$, \\ Julio Cano Loayza ${ }^{1, a}$, Alfredo Castillo Gozzer ${ }^{1, a}$, Jose Luis Castillo Lino ${ }^{1, a}$, Luis Bracamonte Ferrer ${ }^{1, a}$, \\ Xandra Rodriguez Tucto ${ }^{1, a}$, Lorena Manrique ${ }^{1, a}$, Franklin Mendoza ${ }^{1, a}$, Jose Juarez Silva ${ }^{1, a}$, \\ Lourdes Paredes Saravia ${ }^{1, a}$
}

\begin{abstract}
RESUMEN
Objetivo: Comparar el score SOFA al ingreso y a las 24horas, y evaluar su utilidad en la predicción de la muerte materna. Materiales y métodos: Se realizó un estudio descriptivo de tipo transversal de las pacientes admitidas a la UCI Materna del INMP desde agosto del 2014 a Julio del 2019. Se aplicaron pruebas paramétricas o no paramétricas según evaluación previa de normalidad y se usó una significación estadística $p<0.05$. El análisis estadístico se realizó utilizando el paquete estadístico STATA versión 13. Resultados: La media de la edad corresponde a 28.32 años. La media de la edad gestacional correspondió a 31.33 semanas; La media de la estancia hospitalaria fue de 4.52 días. El area bajo la curva para el SOFA al ingreso fue de $0.8818 \mathrm{y}$ el area bajo la curva del SOFA a las 24 horas fue de 0.9755 . $P=0.1225$. No se encontraron diferencias significativas entre ellas. Se determinó que el mejor punto de corte corresponde a un Score de 6 con una sensibilidad que corresponde a $89.29 \%$ y una especificidad de $79.13 \%$. Conclusiones: El Score SOFA se adapta bien a las pacientes obstétricas admitidas en los ambientes de Cuidados Intensivos Materno, manteniendo un corte de 6 para el SOFA de ingreso con una buena sensibilidad y especificidad. (89.29\%, 79.13\%). El Score SOFA es útil para ser usado en ambientes de UCl y además debe sugerirse su uso en toda UCl obstétrica de nuestro país.
\end{abstract}

Palabras claves: Score SOFA; Muerte materna; UCI obstétrica. (Fuente: DeCS BIREME).

\section{USEFULNESS OF THE SOFA SCORE IN THE PREDICTION OF MATERNAL DEATH IN THE MATERNAL ICU OF THE INMP}

\begin{abstract}
Objective: To compare the SOFA score at admission and 24 hours and evaluate its usefulness in predicting maternal death. Materials and methods: A cross-sectional descriptive study of patients admitted to the Maternal ICU of the INMP was carried out from August 2014 to July 2019. Parametric and non-parametric tests were applied according to a previous evaluation of normality and a statistical significance $p<0.05$. Statistical analysis was performed using the statistical package STATA version 13. Results: The mean age corresponds to 28.32 years. The mean gestational age corresponded to 31.33 weeks; The mean hospital stay was 4.52 days. The area under the curve for SOFA at entry was 0.8818 and the area under the curve for SOFA at 24 hours was 0.9755 . $P=0.1225$. No significant differences were found between them. It was determined that the best cut-off point corresponds to a Score of 6 with a sensitivity that corresponds to $89.29 \%$ and a specificity of $79.13 \%$. Conclusions: The SOFA Score adapts well to obstetric patients admitted to Maternal Intensive Care settings, maintaining a cut-off of 6 for admission SOFA with good sensitivity and specificity. $(89.29 \%, 79.13 \%)$. The SOFA score is useful in maternal ICU environments and it should be used in our country.
\end{abstract}

Key words: SOFA Score; Maternal death; Obstetric ICU. (Source: MeSH NLM).

\section{INTRODUCCIÓN}

Los scores pronostico como el APACHE aparecieron el año de 1985 con el objetivo de predecir la mortalidad del paciente en $\mathrm{UCI}^{1,2}$ a través de los años han aparecido una serie de scores pronostico con diferentes objetivos, como predecir la mortalidad, la posibilidad de admisión a UCI, la probabilidad de complicaciones en $\mathrm{UCl}^{3}$. El score SOFA fue desarrollado el año de 1994 durante una conferencia de consenso organizado por la sociedad europea de cuidados intensivos y medicina de emergencia en un intento de proporcionar medios de describir cuantitativa, y objetivamente el grado de falla orgánica tanto en pacientes individuales como en grupo ${ }^{4-6}$. Sin embargo las pacientes admitidas a Unidades de Cuidados intensivos materno no cuenta con scores pronóstico que permitan predecir la mortalidad $u$ cualquier otro evento y para hacerlo se ha intentado usar los scores que hay en el medio ${ }^{7,8}$. Sin embargo, ninguno de ellos predice la mortalidad adecuadamente para las pacientes obstétricas, El score SOFA es el que más se acerca en predecir la mortalidad en la paciente obstétrica. Algunos otros han modificado el score SOFA para mejorar

\footnotetext{
Instituto Nacional Materno Perinatal. Lima, Perú

a Médico Intensivista. Unidad de Cuidados Intensivos Materno.

Citar como: De La peña WJ, Diaz A, Meza R, Sandoval H, Cano J, Castillo A, Castillo JL, Bracamonte L, Rodrigues X, Manrique L, Mendoza F, Juarez J, Paredes L. Utilidad del score sofa en la prediccion de muerte materna en la UCI materna del INMP. Rev Peru Investig Matern Perinat 2020; 9(3):18-22 DOI https://doi.org/10.33421/inmp.2020209
}

Recibido: 13-07-20 Aprobado: 30-09-20 
su predictibilidad sobre la sepsis obstétrica ${ }^{5,9,10}$. El presente trabajo pretende evaluar el score SOFA en la predicción de la mortalidad materna para cualquiera de sus causas, así como determinar el mejor punto de corte para la mejor sensibilidad y especificidad. Se pretende además comparar el score SOFA al ingreso y a las 24 horas, evaluar la utilidad del Score SOFA al ingreso y a las 24 Horas en la predicción de la muerte materna, determinar el punto de corte para para la predicción de muerte materna usando el score SOFA de ingreso, determinar el punto de corte para la predicción de muerte materna usando el score SOFA a las 24 horas, determinar el área bajo la curva del score SOFA de ingreso y de 24 horas. Se utilizara el punto de corte hallado para evaluar las medias de las variables cuantitativas y las variables categóricas.

\section{MATERIALES Y MÉTODOS}

Se realizó un estudio descriptivo de tipo transversal. Se estudiaron pacientes admitidas a la Unidad de Cuidados Intensivos (UCl) Materna del Instituto Nacional Materno Perinatal (INMP) desde agosto del 2014 a Julio del 2019.

Se incluyeron al estudio las pacientes admitidas a la UCI Materna del INMP de agosto del 2014 a Julio del 2019, con diagnóstico de complicaciones asociadas al embarazo. Por lo tanto, se excluyeron del estudio aquellas pacientes que ingresaron por causas distintas al embarazo. Se consideraron todas las pacientes admitidas a la UCI Materna en el periodo de estudio con criterios de inclusión; muestra por conveniencia. Se determinó el Área bajo la curva del Score SOFA de pacientes admitidas a la UCI Materna con criterio de inclusión que registraron en la base de datos (base de datos de la UCI Materna del INMP en EPIINFO versión 7) el registro de SOFA de ingreso y SOFA a las 24 horas.
En el análisis estadístico, para las variables cuantitativas edad, número de gestaciones, edad gestacional. Las variables cuantitativas previa evaluación de normalidad se compararon obteniendo el $\mathrm{p}$ valor, las variables cualitativas se compararon mediante tablas de frecuencia. Se aplicaron pruebas paramétricas o no paramétricas según evaluación previa de normalidad y se usó una significación estadística $p<0.05$. Se utilizó el paquete estadístico STATA versión 13.

Al ser un estudio transversal de tipo descriptivo, no contraviene las normas de ética relacionados a investigación en salud.

\section{RESULTADOS}

Se admitieron al estudio un total de 363 pacientes. La media de la edad de las fue de 28.32 años donde el valor mínimo fue de 14 años y una edad máxima de 46 años. La media de la edad gestacional correspondió a 31.33 semanas; con un mínimo de 4 y la máxima de 42 semanas. La media de la estancia hospitalaria fue de 4.52 días con un valor mínimo de 1 y un máximo de 92 días. Se comparó el area bajo la curva del Score SOFA de pacientes admitidas a la UCI Materna del INMP tanto al ingreso como a las 24 horas. El area bajo la curva para el SOFA al ingreso fue de 0.8818 y el area bajo la curva del SOFA a las 24 horas fue de 0.9755 .

Al comparar el Score SOFA de ingreso y a las 24 horas observamos que el $\mathrm{P}$ valor es de 0.1225 ( $p>0.05)$ por lo tanto se acepta la hipótesis nula. El área bajo la curva del SOFA al ingreso y a las 24 horas son iguales (Figura 1).

Cuando se evalúa el Score SOFA al ingreso de manera independiente se observa se determina que el mejor punto

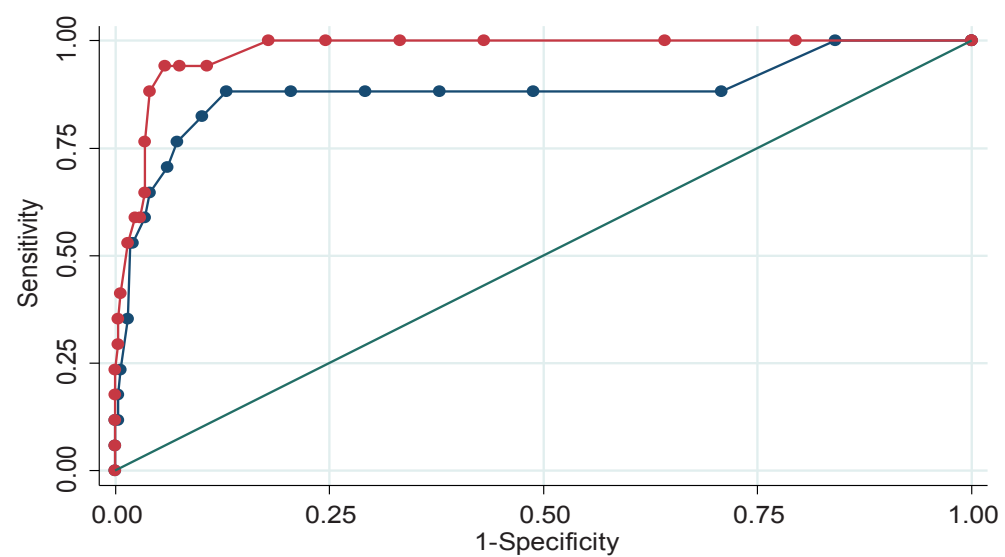

\begin{tabular}{lcccc|}
\hline $\begin{array}{l}\longrightarrow \\
- \\
\text { Refaaling ROC area: } \\
\text { Reference }\end{array}$ & 0.8818 & - & & \\
\hline & OBS & AREA & ES & IC 95\% \\
\hline SOFA ING & 363 & 0.8818 & 0.0607 & $0.76-1.00$ \\
\hline SOFA 24HORAS & 363 & 0.9755 & 0.0098 & $0.95-0.99$ \\
\hline
\end{tabular}

Figura 1. Curva ROC para score SOFA al ingreso y a las 24 horas y su área bajo la curva 
Tabla 1. Sensibilidad y especificidad del Score SOFA al ingreso según puntos de corte

\begin{tabular}{|c|c|c|c|c|c|}
\hline Punto de corte & Sensibilidad & Especificidad & Clasif & LR+ & LR- \\
\hline$(>=0)$ & $100.00 \%$ & $0.00 \%$ & $6.03 \%$ & 1.0000 & \\
\hline$(>=1)$ & $100.00 \%$ & $16.06 \%$ & $21.12 \%$ & 1.1913 & 0.0000 \\
\hline$(>=2)$ & $92.86 \%$ & $31.65 \%$ & $35.34 \%$ & 1.3586 & 0.2257 \\
\hline$(>=3)$ & $92.86 \%$ & $50.92 \%$ & $53.45 \%$ & 1.8919 & 0.1403 \\
\hline$(>=4)$ & $92.86 \%$ & $62.16 \%$ & $64.01 \%$ & 2.4537 & 0.1149 \\
\hline$(>=5)$ & $92.86 \%$ & $71.10 \%$ & $72.41 \%$ & 3.2132 & 0.1005 \\
\hline$(>=6)$ & $89.29 \%$ & $79.13 \%$ & $79.74 \%$ & 4.2779 & 0.1354 \\
\hline$(>=7)$ & $89.29 \%$ & $86.01 \%$ & $86.21 \%$ & 6.3817 & 0.1246 \\
\hline$(>=8)$ & $82.14 \%$ & $89.22 \%$ & $88.79 \%$ & 7.6201 & 0.2001 \\
\hline$(>=9)$ & $78.57 \%$ & $92.43 \%$ & $91.59 \%$ & 10.3809 & 0.2318 \\
\hline$(>=10)$ & $71.43 \%$ & $93.58 \%$ & $92.24 \%$ & 11.1224 & 0.3053 \\
\hline$(>=11)$ & $64.29 \%$ & $95.87 \%$ & $93.97 \%$ & 15.5714 & 0.3725 \\
\hline$(>=12)$ & $57.14 \%$ & $96.33 \%$ & $93.97 \%$ & 15.5714 & 0.4449 \\
\hline$(>=13)$ & $46.43 \%$ & $97.71 \%$ & $94.61 \%$ & 20.2428 & 0.5483 \\
\hline$(>=14)$ & $42.86 \%$ & $97.94 \%$ & $94.61 \%$ & 20.7619 & 0.5835 \\
\hline$(>=15)$ & $32.14 \%$ & $98.17 \%$ & $94.18 \%$ & 17.5179 & 0.6913 \\
\hline$(>=16)$ & $25.00 \%$ & $99.31 \%$ & $94.83 \%$ & 36.3330 & 0.7552 \\
\hline$(>=17)$ & $14.29 \%$ & $99.77 \%$ & $94.61 \%$ & 62.2865 & 0.8591 \\
\hline$(>=18)$ & $10.71 \%$ & $99.77 \%$ & $94.40 \%$ & 46.7149 & 0.8949 \\
\hline$(>=19)$ & $7.14 \%$ & $99.77 \%$ & $94.18 \%$ & 31.1433 & 0.9307 \\
\hline$(>=20)$ & $7.14 \%$ & $100.00 \%$ & $94.40 \%$ & & 0.9286 \\
\hline$(>=24)$ & $3.57 \%$ & $100.00 \%$ & $94.18 \%$ & & 0.9643 \\
\hline$(>24)$ & $0.00 \%$ & $100.00 \%$ & $93.97 \%$ & & 1.0000 \\
\hline
\end{tabular}

de corte corresponde a un Score de 6 con una sensibilidad que corresponde a $89.29 \%$ y una especificidad de $79.13 \%$; en comparación al Score SOFA a las 24horas, donde el puntaje de14, corresponde a $93.75 \%$ y una especificidad de $84.83 \%$ (Tabla 1 y 2 ).

Al evaluar las variables cuantitativas (Tabla 3 ) comparadas en dos grupos generados por el punto de corte de 6 de score SOFA. Se encuentra que las medias de ambos grupos no presentan diferencias significativas $(p=0.129)$, al evaluar la variable estancia hospitalaria las diferencias de las medias presentan diferencias significativas ( $p=0.0003$ ), al evaluar las medias de la variable índice de masa corporal (IMC) se encuentra diferencias significativas $(p=0.0233)$, al evaluar las medias de la variable edad gestacional (EG) no se encontraron diferencias estadísticas significativas

Tabla 2. Sensibilidad y especificidad del score SOFA a las 24 horas según puntos de corte.

\begin{tabular}{|c|c|c|c|c|c|}
\hline Punto de corte & Sensibilidad & Especificidad & Classified & LR+ & LR- \\
\hline$(>=0)$ & $100.00 \%$ & $0.00 \%$ & $7.05 \%$ & 1.0000 & \\
\hline$(>=1)$ & $100.00 \%$ & $7.11 \%$ & $13.66 \%$ & 1.0765 & 0.0000 \\
\hline$(>=2)$ & $100.00 \%$ & $11.85 \%$ & $18.06 \%$ & 1.1344 & 0.0000 \\
\hline$(>=3)$ & $100.00 \%$ & $12.80 \%$ & $18.94 \%$ & 1.1467 & 0.0000 \\
\hline$(>=4)$ & $100.00 \%$ & $14.22 \%$ & $20.26 \%$ & 1.1657 & 0.0000 \\
\hline$(>=5)$ & $100.00 \%$ & $15.64 \%$ & $21.59 \%$ & 1.1854 & 0.0000 \\
\hline$(>=6)$ & $100.00 \%$ & $18.96 \%$ & $24.67 \%$ & 1.2339 & 0.0000 \\
\hline$(>=8)$ & $100.00 \%$ & $19.43 \%$ & $25.11 \%$ & 1.2412 & 0.0000 \\
\hline$(>=9)$ & $100.00 \%$ & $20.85 \%$ & $26.43 \%$ & 1.2635 & 0.0000 \\
\hline$(>=10)$ & $100.00 \%$ & $21.33 \%$ & $26.87 \%$ & 1.2711 & 0.0000 \\
\hline$(>=13)$ & $100.00 \%$ & $83.89 \%$ & $85.02 \%$ & 6.2059 & 0.0000 \\
\hline$(>=14)$ & $93.75 \%$ & $84.83 \%$ & $85.46 \%$ & 6.1816 & 0.0737 \\
\hline$(>=15)$ & $93.75 \%$ & $85.31 \%$ & $85.90 \%$ & 6.3810 & 0.0733 \\
\hline$(>=18)$ & $87.50 \%$ & $87.68 \%$ & $87.67 \%$ & 7.1010 & 0.1426 \\
\hline$(>=20)$ & $87.50 \%$ & $88.15 \%$ & $88.11 \%$ & 7.3850 & 0.1418 \\
\hline$(>=21)$ & $81.25 \%$ & $91.47 \%$ & $90.75 \%$ & 9.5243 & 0.2050 \\
\hline$(>=22)$ & $81.25 \%$ & $91.94 \%$ & $91.19 \%$ & 10.0846 & 0.2039 \\
\hline$(>=25)$ & $81.25 \%$ & $92.42 \%$ & $91.63 \%$ & 10.7148 & 0.2029 \\
\hline$(>=26)$ & $81.25 \%$ & $92.89 \%$ & $92.07 \%$ & 11.4292 & 0.2018 \\
\hline
\end{tabular}


Tabla 3. Variables cuantitativas según puntaje SOFA al ingreso

\begin{tabular}{|c|c|c|c|c|c|c|c|c|c|}
\hline \multirow{2}{*}{ VARIABLES } & \multicolumn{4}{|c|}{ SOFA $<6$} & \multicolumn{4}{|c|}{ SOFA $\geq 6$} & \multirow{2}{*}{$\mathbf{P}$} \\
\hline & $\mathbf{N}$ & MEDIA & DE & IC95\% & $\mathbf{N}$ & MEDIA & DE & IC $95 \%$ & \\
\hline Edad & 344 & 27.78 & 7.44 & $26.99-28.57$ & 112 & 29.10 & 7.75 & $7.65-30.55$ & 0.129 \\
\hline Estancia & 261 & 3.51 & 3.00 & $3.15-3.88$ & 71 & 10.30 & 15.57 & $6.81-13.79$ & 0.0003 \\
\hline IMC & 300 & 25.82 & 6.51 & $25.08-26.56$ & 82 & 28.15 & 6.74 & $26.67-29.63$ & 0.0233 \\
\hline EG & 298 & 30.61 & 8.37 & $29.65-31.56$ & 90 & 30.73 & 6.58 & $29.35-3$ & 0.356 \\
\hline Peso P & 234 & 2336.19 & 1086.38 & $2197.27-2476.12$ & 63 & 2061.81 & 1025.34 & $1803.58-2320.03$ & 0.072 \\
\hline Talla P & 218 & 66.63 & 98.43 & $53.49-79.77$ & 54 & 15.16 & 111.41 & $46.44-107.26$ & 0.727 \\
\hline $\mathrm{HB}$ & 321 & 8.89 & 8.11 & $8.00-9.78$ & 102 & 7.55 & 2.35 & 7.09-8.02 & 0.0092 \\
\hline Glucosa & 300 & 126.39 & 61.91 & $119.35-133.42$ & 97 & 155.13 & 72.86 & $140.44-169.81$ & 0.0000 \\
\hline TGO & 294 & 163.17 & 399.74 & $117.29-209.06$ & 97 & 698.16 & 1296.14 & 436.93-959.39 & 0.0000 \\
\hline TGP & 295 & 140.96 & 376.06 & $97.88-184.05$ & 97 & 486.69 & 901.83 & $304.921-668.42$ & 0.0000 \\
\hline BT & 296 & 2.14 & 4.20 & $1.66-2.62$ & 95 & 4.90 & 6.07 & $3.66-6.14$ & 0.0000 \\
\hline Albumina & 221 & 2.17 & 1.85 & $1.92-2.42$ & 85 & 2.10 & 1.74 & $1.72-2.47$ & 0.0746 \\
\hline Fibrinogeno & 279 & 355.57 & 136.51 & $339.48-371.66$ & 92 & 304.94 & 56.041 & $272.63-337.26$ & 0.0019 \\
\hline
\end{tabular}

$(p=0.356)$, al evaluar las medias del peso de los productos no se encontraron diferencias significativas $(p=0.072)$, al evaluar las medias de la Talla de los productos no se encontraron diferencias significativas $(p=0.727)$, al evaluar las medias de la variable hemoglobina $(\mathrm{Hb})$ se encontraron diferencias estadísticas significativas $(p=0.0092)$, al evaluar las medias de la variable glucosa se encontraron diferencias estadísticas significativas $(p=0.0000)$, al evaluar las medias de la variable TGO se observó diferencias significativas $(p=0.0000)$, al evaluar las medias de la variable TGP se encontraron diferencias significativas $(p=0.0000)$, al evaluar las medias de la variable bilirrubina total (BT) se encontraron diferencias significativas $(p=0.0000)$, al evaluar las medias de la variable albumina no se encontraron diferencias estadísticas significativas ( $p=0.0746)$, al evaluar las medias de la variable fibrinógeno se encontraron diferencias estadísticas significativas $(p=0.0019)$.

Se evaluaron las variables categóricas comparadas con el punto de corte hallado para el score SOFA que fue de 6 (Tabla 4). Para la variable edad categorizada por grupos nos e encontraron diferencias estadísticas significativas ( $p=0.633$ ), al evaluar el índice de masa corporal (IMC) no se encontraron diferencias estadísticas significativas $(p=0.160)$, al evaluar la variable estado civil no se encontraron diferencias significativas $(p=0.116)$, al evaluar el grado de instrucción no se encontraron diferencias significativas $(p=0.747)$

Tabla 4. Variables cualitativas según score SOFA al ingreso

\begin{tabular}{|c|c|c|c|c|c|}
\hline & \multicolumn{2}{|c|}{ SOFA $<6$} & \multicolumn{2}{|c|}{ SOFA $\geq 6$} & \multirow{2}{*}{$\mathbf{P}$} \\
\hline & $\mathrm{N}$ & $\%$ & $\mathrm{~N}$ & $\%$ & \\
\hline$\overline{\text { EDAD (años) }}$ & & & & & 0.633 \\
\hline$<20$ & 73 & 16 & 19 & 4.16 & \\
\hline $21-30$ & 145 & 31.79 & 46 & 10.08 & \\
\hline $31-39$ & 108 & 23.68 & 39 & 8.55 & \\
\hline$>40$ & 18 & 3.94 & 8 & 1.75 & \\
\hline IMC & $\mathbf{N}$ & $\%$ & $\mathbf{N}$ & $\%$ & 0.160 \\
\hline Bajo peso & 9 & 2.42 & 1 & 0.26 & \\
\hline Normal & 129 & 34.67 & 25 & 6.72 & \\
\hline Sobrepeso & 107 & 28.76 & 34 & 9.13 & \\
\hline Obesidad I & 38 & 10.21 & 3 & 0.81 & \\
\hline Obesidad II & 13 & 3.49 & 6 & 1.61 & \\
\hline Obesidad III & 4 & 1.07 & 3 & 0.81 & \\
\hline ESTADO CIVIL & & & & & 0.116 \\
\hline Casada & 41 & 8.77 & 24 & 5.13 & \\
\hline Conviviente & 233 & 49.89 & 69 & & \\
\hline Soltera & 70 & 14.98 & 25 & & \\
\hline Viuda & 4 & 0.85 & 1 & & \\
\hline GRADO DE INSTRUCCIÓN & & & & & 0.747 \\
\hline Primaria & 50 & 10.82 & 17 & 3.67 & \\
\hline Secundaria & 240 & 51.94 & 75 & 16.23 & \\
\hline Sin instrucción & 2 & 0.43 & 2 & 0.43 & \\
\hline Superior & 39 & 8.44 & 14 & 3.03 & \\
\hline Técnico & 17 & 3.67 & 6 & 1.29 & \\
\hline
\end{tabular}




\section{DISCUSIÓN}

La necesidad de contar con un score que permita predecir cualquier evento de interés en la $\mathrm{UCI}$ obstétrica; admisión a $\mathrm{UCl}$, complicaciones en $\mathrm{UCl}$, Sepsis, y mortalidad materna, es aún una deuda de la investigación médica en el ámbito de los cuidados críticos obstétricos. Mientras no se cuente con un SCORE que se construya desde la visión de la medicina critica obstétrica el Score SOFA con cortes adaptados a la realidad local nos permitirán tener un valor cuantitativo que permita predecir la mortalidad materna en UCI así como identificar a las pacientes obstétricas con riesgo de muerte a las cuales se les brinde la atención adecuada que permita la solución a sus problemas médicos de tal forma de reducir la morbimortalidad materna institucional y sugerir su uso a nivel nacional.

En conclusión, la evaluación del Score SOFA para pacientes admitidas a la UCl del INMP ha permitido determinar que el Score SOFA tanto del ingreso, así como a las 24 horas son buenas pruebas diagnósticas para predecir la mortalidad materna en la $\mathrm{UCl}$. Se requieren mayores estudios para determinar cuáles son las disfunciones principales que aportan mayor porcentaje al score SOFA total. Asimismo, es necesario crear un Score SOFA modificado que permita mejorar la predictibilidad y el valor predictivo positivo en este grupo de pacientes.

Se debe además comparar los scores existentes, los modificados, así como los nuevos scores para determinar cuál de ellos es el que mejor predictibilidad aporten, el hallazgo del presente estudio nos permite afirmar que el Score SOFA se adapta muy bien a las pacientes obstétricas admitidas en los ambientes de Cuidados Intensivos Materno, manteniendo un corte de 6 para el SOFA de ingreso con una buena sensibilidad $y$ especificidad. $(89.29 \%, 79.13 \%)$. Como se ha determinado en otros estudios de investigación el Score SOFA es útil para ser usado en ambientes de $\mathrm{UCl}$ y además debe sugerirse su uso en toda $\mathrm{UCl}$ obstétrica de nuestro país.

Financiamiento: Autofinanciado.

Conflicto de interés: Los autores declaran no tener algún conflicto de intereses

\section{REFERENCIAS BIBLIOGRÁFICAS}

1. APACHE II: a severity of disease classification system. - PubMed - NCBI [Internet]. [citado 7 de octubre de 2019]. Disponible en: https://www.ncbi.nlm.nih.gov/ pubmed/3928249

2. Safari S, Shojaee M, Rahmati F, Barartloo A, Hahshemi B, Forouzanfar MM, et al. Accuracy of SOFA score in prediction of 30-day outcome of critically ill patients. Turk J Emerg Med. diciembre de 2016;16(4):146-50.

3. Esquivel LAB, Urbina JM, Zerón HM. Approach to an obstetric prognosis scale: The modified SOFA scale. 2016;50(3):7.

4. SOFA Score - an overview | ScienceDirect Topics [Internet]. [citado 28 de agosto de 2019]. Disponible en: https://www. sciencedirect.com/topics/medicine-and-dentistry/sofa-score

5. Khwannimit B, Bhurayanontachai R, Vattanavanit V. Comparison of the accuracy of three early warning scores with SOFA score for predicting mortality in adult sepsis and septic shock patients admitted to intensive care unit. Heart Lung J Crit Care. junio de 2019;48(3):240-4.

6. Lee KS, Sheen SS, Jung YJ, Park RW, Lee YJ, Chung WY, et al. Consideration of additional factors in Sequential Organ Failure Assessment score. J Crit Care. febrero de 2014;29(1):185.e9-185.e12.

7. Gunes Ozaydin M, Guneysel O, Saridogan F, Ozaydin V. Are scoring systems sufficient for predicting mortality due to sepsis in the emergency department? Turk J Emerg Med. 5 de diciembre de 2016;17(1):25-8.

8. Hewett JN, Rodgers GW, Chase JG, Le Compte AJ, Pretty CG, Shaw GM. Assessment of SOFA Score as a Diagnostic Indicator in Intensive Care Medicine. IFAC Proc Vol. 1 de enero de 2012;45(18):467-72.

9. Vincent J-L, Moreno R, Takala J, Willatts S, De Mendonça A, Bruining $\mathrm{H}$, et al. The SOFA (Sepsis-related Organ Failure Assessment) score to describe organ dysfunction/failure. Intensive Care Med. 1 de julio de 1996;22(7):707-10.

10. Songsangjinda T, Khwannimit B. Comparison of severity score models based on different sepsis definitions to predict inhospital mortality among sepsis patients in the Intensive Care Unit. Med Intensiva [Internet]. [citado 30 de agosto de 2019]; Disponible en: http://www.medintensiva.org/es-comparisonseverity-score-models-based-articulo-S0210569118303371

\section{Correspondencia:}

Walter J. De la Peña Meniz

Dirección: Unidad de Cuidados Intensivos Materno.

Instituto Nacional Materno Perinatal. Jr. Santa Rosa, 941.

Cercado de Lima, Lima, Perú

Teléfono: 996014918 